PROCEEDINGS OF THE

AMERICAN MATHEMATICAL SOCIETY

Volume 131, Number 2, Pages 511-521

S 0002-9939(02)06586-3

Article electronically published on June 3, 2002

\title{
THE $\ell^{1}$-INDICES OF TSIRELSON TYPE SPACES
}

\author{
DENNY H. LEUNG AND WEE-KEE TANG
}

(Communicated by N. Tomczak-Jaegermann)

\begin{abstract}
If $\alpha$ and $\beta$ are countable ordinals such that $\beta \neq 0$, denote by $\widetilde{T}_{\alpha, \beta}$ the completion of $c_{00}$ with respect to the implicitly defined norm

$$
\|x\|=\max \left\{\|x\|_{\mathcal{S}_{\alpha}}, \frac{1}{2} \sup \sum_{i=1}^{j}\left\|E_{i} x\right\|\right\},
$$

where the supremum is taken over all finite subsets $E_{1}, \ldots, E_{j}$ of $\mathbb{N}$ such that $E_{1}<\cdots<E_{j}$ and $\left\{\min E_{1}, \ldots, \min E_{j}\right\} \in \mathcal{S}_{\beta}$. It is shown that the Bourgain $\ell^{1}$-index of $\widetilde{T}_{\alpha, \beta}$ is $\omega^{\alpha+\beta \cdot \omega}$. In particular, if $\omega_{1}>\alpha=\omega^{\alpha_{1}} \cdot m_{1}+\cdots+\omega^{\alpha_{n}} \cdot m_{n}$ in Cantor normal form and $\alpha_{n}$ is not a limit ordinal, then there exists a Banach space whose $\ell^{1}$-index is $\omega^{\alpha}$.
\end{abstract}

Let $E$ be a separable Banach space not containing a copy of $\ell^{1}$. There are several measures of the complexity of $\ell^{1}(n)$ 's inside $E$. These include the Bourgain $\ell^{1}$-index [4], the existence of so-called $\ell_{\alpha}^{1}$-spreading models ([2] and [9]) and the asymptotic constants as defined by Odell, Tomczak-Jaegermann, and Wagner [11. In this paper, we concentrate on the first two measures in Tsirelson type spaces. It is easy to see that the existence of $\ell_{\alpha}^{1}$-spreading models implies a large $\ell^{1}$-index. In general, the implication is not reversible [8, Remark 6.6(i)]. However, suppose that $T$ is the standard Tsirelson space constructed by Figiel and Johnson [6] (the dual of the original Tsirelson space [12]). It is known that there is a constant $K$ such that every normalized block basic sequence in $T$ is $K$-equivalent to a subsequence of the unit vector basis of $T$ (see e.g., 5 ). Using this observation, one can show that the existence of $\ell^{1}$-block trees in $T$ with large indices leads to the existence of large $\ell_{\alpha}^{1}$-spreading models. The result can be used to calculate the $\ell^{1}$-index of $T$. In this paper, we show that a similar method can be applied to certain general Tsirelson type spaces. In particular, it is shown that if $\omega_{1}>\alpha=\omega^{\alpha_{1}} \cdot m_{1}+\cdots+\omega^{\alpha_{n}} \cdot m_{n}$ in Cantor normal form and $\alpha_{n}$ is not a limit ordinal, then there exists a Banach space whose $\ell^{1}$-index is $\omega^{\alpha}$. This gives a partial answer to Question 1 in 8 . The authors have extended the method to compute the Bourgain $\ell^{1}$-indices of mixed Tsirelson spaces [10]. In particular, it is shown there that if $\alpha$ is not of the form $\omega^{\beta}$ for some limit ordinal $\beta$, then there exists a Banach space whose $\ell^{1}$-index is $\omega^{\alpha}$.

If $M$ is an infinite subset of $\mathbb{N}$, denote the set of all finite, respectively infinite, subsets of $M$ by $[M]^{<\infty}$, respectively $[M]$. A subset $\mathcal{F}$ of $[\mathbb{N}]^{<\infty}$ is hereditary if $G \in \mathcal{F}$ whenever $G \subseteq F \in \mathcal{F} . \mathcal{F}$ is spreading if whenever $F=\left\{n_{1}, \ldots, n_{k}\right\} \in \mathcal{F}$ with $n_{1}<\cdots<n_{k}$ and $m_{1}<\cdots<m_{k}$ satisfies $m_{i} \geq n_{i}$ for $1 \leq i \leq k$, then

Received by the editors March 7, 2001 and, in revised form, July 10, 2001 and September 20, 2001.

2000 Mathematics Subject Classification. Primary 46B20; Secondary 05C05. 
$\left\{m_{1}, \ldots, m_{k}\right\} \in \mathcal{F} . \mathcal{F}$ is compact if it is compact in the product topology in $2^{\mathbb{N}}$. A set $\mathcal{F}$ of finite subsets of $\mathbb{N}$ is called regular if it has all three properties. If $E$ and $F$ are finite subsets of $\mathbb{N}$, we write $E<F$, respectively $E \leq F$, to mean $\max E<\min F$, respectively $\max E \leq \min F(\max \emptyset=0$ and $\min \emptyset=\infty)$. We abbreviate $\{n\}<E$ and $\{n\} \leq E$ to $n<E$ and $n \leq E$ respectively. Given $\mathcal{F} \subseteq[\mathbb{N}]<\infty$, a sequence of finite subsets $\left\{E_{1}, \ldots, E_{n}\right\}$ of $\mathbb{N}$ is said to be $\mathcal{F}$-admissible if $E_{1}<\cdots<E_{n}$ and $\left\{\min E_{1}, \ldots, \min E_{n}\right\} \in \mathcal{F}$. If $\mathcal{M}$ and $\mathcal{N}$ are regular subsets of $[\mathbb{N}]^{<\infty}$, we let

$$
\mathcal{M}[\mathcal{N}]=\left\{\bigcup_{i=1}^{k} F_{i}: F_{i} \in \mathcal{N} \text { for all } i \text { and }\left\{F_{1}, \ldots, F_{k}\right\} \mathcal{M} \text {-admissible }\right\}
$$

and

$$
(\mathcal{M}, \mathcal{N})=\{M \cup N: M<N, M \in \mathcal{M} \text { and } N \in \mathcal{N}\} .
$$

We also write $\mathcal{M}^{2}$ for $(\mathcal{M}, \mathcal{M})$. Of primary importance are the Schreier classes as defined in 1]. Let $\mathcal{S}_{0}=\{\{n\}: n \in \mathbb{N}\} \cup\{\emptyset\}$ and $\mathcal{S}_{1}=\{F \subseteq \mathbb{N}:|F| \leq \min F\}$. Here $|F|$ denotes the cardinality of $F$. The higher Schreier classes are defined inductively as follows. $\mathcal{S}_{\alpha+1}=\mathcal{S}_{1}\left[\mathcal{S}_{\alpha}\right]$ for all $\alpha<\omega_{1}$. If $\alpha$ is a countable limit ordinal, choose a sequence $\left(\alpha_{n}\right)$ strictly increasing to $\alpha$ and set

$$
\mathcal{S}_{\alpha}=\left\{F: F \in \mathcal{S}_{\alpha_{n}} \text { for some } n \leq|F|\right\} .
$$

It is clear that $\mathcal{S}_{\alpha}$ is a regular family for all $\alpha<\omega_{1}$. If $M=\left(m_{1}, m_{2}, \ldots\right)$ is a subsequence of $\mathbb{N}$, let $\mathcal{S}_{\alpha}(M)=\left\{\left\{m_{i}: i \in F\right\}: F \in \mathcal{S}_{\alpha}\right\}$. Since $\mathcal{S}_{\alpha}$ is spreading, $\mathcal{S}_{\alpha}(M) \subseteq \mathcal{S}_{\alpha}$

Let $c_{00}$ be the space of all finitely supported sequences. If $F \in[\mathbb{N}]^{<\infty}$ and $a=\left(a_{n}\right) \in c_{00}$, let $F a=\left(b_{n}\right) \in c_{00}$, where $b_{n}=a_{n}$ if $n \in F$ and 0 otherwise; also set $\sigma_{F}\left(\left(a_{n}\right)\right)=\sum_{n \in F}\left|a_{n}\right|$. Finally, if $\mathcal{S}_{0} \subseteq \mathcal{F} \subseteq[\mathbb{N}]^{<\infty}$, define an associated norm $\|\cdot\|_{\mathcal{F}}$ on $c_{00}$ by $\left\|\left(a_{n}\right)\right\|_{\mathcal{F}}=\sup _{F \in \mathcal{F}} \sigma_{F}\left(\left(a_{n}\right)\right)$.

Definition 1. Let $\alpha, \beta$ be countable ordinals such that $\beta \neq 0$. Define $\|\cdot\|_{n}$ and $\|\cdot\|_{n}^{\prime}, n \in \mathbb{N}$, inductively as follows. Let $\|\cdot\|_{0}=\|\cdot\|_{0}^{\prime}=\|\cdot\|_{\mathcal{S}_{\alpha}}$. If $x \in c_{00}$, set

$$
\|x\|_{n+1}=\max \left\{\|x\|_{n}, \sup \left\{\frac{1}{2} \sum_{i=1}^{j}\left\|E_{i} x\right\|_{n}:\left\{E_{1}, \ldots, E_{j}\right\} \mathcal{S}_{\beta} \text {-admissible }\right\}\right\}
$$

and

$$
\|x\|_{n+1}^{\prime}=\max \left\{\|x\|_{n}^{\prime}, \sup \left\{\frac{1}{2} \sum_{i=1}^{j}\left\|E_{i} x\right\|_{n}^{\prime}:\left\{E_{1}, \ldots, E_{j}\right\} \quad\left(\mathcal{S}_{\beta}\right)^{2} \text {-admissible }\right\}\right\} .
$$

Note that $\left(\|x\|_{n}\right)_{n \in \mathbb{N}}$ and $\left(\|x\|_{n}^{\prime}\right)_{n \in \mathbb{N}}$ are increasing sequences majorized by the $\ell^{1}$-norm of $x$. Let $\|x\|_{\widetilde{T}}=\lim _{n \rightarrow \infty}\|x\|_{n}$ and $\|x\|_{\tilde{T}}=\lim _{n \rightarrow \infty}\|x\|_{n}^{\prime}$. Denote by $\tilde{T}_{\alpha, \beta}$ and $\widetilde{\widetilde{T}}_{\alpha, \beta}$ respectively the completion of $c_{00}$ under the norms $\|\cdot\|_{\widetilde{T}}$ and $\|\cdot\|_{\widetilde{T}}$. Clearly, $\widetilde{T}_{0,1}$ is the Tsirelson space constructed by Figiel and Johnson [6] and $\widetilde{T}_{0, \beta}$ is the space denoted by $T\left(\mathcal{S}_{\beta}, \frac{1}{2}\right)$ in $\left[8\right.$. The $\ell^{1}$-index of $\tilde{T}_{0, \beta}$ is shown to be $\omega^{\beta \cdot \omega}$ in $[8]$. Here, we use a different argument to compute the $\ell^{1}$-indices of the spaces $\widetilde{T}_{\alpha, \beta}$. The next proposition can be verified immediately. 
Proposition 2. The norms $\|\cdot\|_{\widetilde{T}}$ and $\|\cdot\|_{\widetilde{T}}$ satisfy the implicit equations

$$
\|x\|_{\widetilde{T}}=\max \left\{\|x\|_{\mathcal{S}_{\alpha}}, \sup \left\{\frac{1}{2} \sum_{i=1}^{j}\left\|E_{i} x\right\|_{\widetilde{T}}:\left\{E_{1}, \ldots, E_{j}\right\} \mathcal{S}_{\beta} \text {-admissible }\right\}\right\}
$$

and

$$
\|x\|_{\widetilde{T}}=\max \left\{\|x\|_{\mathcal{S}_{\alpha}}, \sup \left\{\frac{1}{2} \sum_{i=1}^{j}\left\|E_{i} x\right\|_{\widetilde{T}}:\left\{E_{1}, \ldots, E_{j}\right\}\left(\mathcal{S}_{\beta}\right)^{2} \text {-admissible }\right\}\right\}
$$

for all $x \in c_{00}$.

Proposition 4 is a close relative of Lemma 5 in [5. It is the key observation that allows us to reduce $\ell^{1}$-block trees on $\widetilde{T}_{\alpha, \beta}$ to subsequences of the unit vector basis $\left(e_{n}\right)$ of $\widetilde{T}_{\alpha, \beta}$. The following lemma is easily established by induction.

Lemma 3. Suppose that $n_{1} \leq I_{1}<n_{2} \leq I_{2}<\ldots<n_{k} \leq I_{k}$ and $\left|I_{j}\right| \leq 2$ for $1 \leq j \leq k$. If $\left\{n_{1}, n_{2}, \ldots, n_{k}\right\} \in \mathcal{S}_{\beta}$ for some $\beta<\omega_{1}$, then $\bigcup_{j=1}^{k} I_{j} \in\left(\mathcal{S}_{\beta}\right)^{2}$.

Obviously, the sequence of coordinate unit vectors $\left(u_{n}\right)$ forms a normalized 1unconditional basis of $\widetilde{T}_{\alpha, \beta}$. The support of an element $x=\sum a_{n} u_{n} \in \tilde{T}_{\alpha, \beta}$ is the set $\operatorname{supp} x=\left\{n: a_{n} \neq 0\right\}$.

Proposition 4. For every $\|\cdot\|_{\widetilde{T}}$-normalized block basis $\left(x_{1}, x_{2}, \ldots, x_{p}\right)$ in $\tilde{T}_{\alpha, \beta}$ and all $\left(a_{k}\right) \in c_{00}$,

$$
\left\|\sum_{k=1}^{p} a_{k} x_{k}\right\|_{\widetilde{T}} \leq 2\left\|\sum_{k=1}^{p} a_{k} e_{i_{k}}\right\|_{\widetilde{T}}
$$

where $i_{k}=\max \operatorname{supp} x_{k}$, and $\left(e_{n}\right)$ is the unit vector basis of $\widetilde{\widetilde{T}}_{\alpha, \beta}$.

Proof. With the notation as above, we prove by induction that $\left\|\sum_{k=1}^{p} a_{k} x_{k}\right\|_{n} \leq$ $2\left\|\sum_{k=1}^{p} a_{k} e_{i_{k}}\right\|_{n}^{\prime}$ for all $n \in \mathbb{N} \cup\{0\},\left(a_{k}\right) \in c_{00}$. To establish the inequality for the case $n=0$, let $I \in \mathcal{S}_{\alpha}$. Define $J=\left\{k: I \cap \operatorname{supp} x_{k} \neq \emptyset\right\}$. Then

$$
\begin{aligned}
\sigma_{I}\left(\sum_{k=1}^{p} a_{k} x_{k}\right) & =\sum_{k=1}^{p}\left|a_{k}\right| \sigma_{I}\left(x_{k}\right) \\
& \leq \sum_{k \in J}\left|a_{k}\right|\left\|x_{k}\right\|_{0} \\
& \leq \sum_{k \in J}\left|a_{k}\right|=\sigma_{L}\left(\sum_{k=1}^{p} a_{k} e_{i_{k}}\right), \text { where } L=\left\{i_{k}: k \in J\right\}, \\
& \leq\left\|\sum_{k=1}^{p} a_{k} e_{i_{k}}\right\|_{0}^{\prime}, \text { since } L \in \mathcal{S}_{\alpha} .
\end{aligned}
$$

Suppose the proposition holds for some $n$. Let $\left\{E_{1}, \ldots, E_{q}\right\}$ be $\mathcal{S}_{\beta}$-admissible. Without loss of generality, we may assume that $E_{1}, \ldots, E_{q}$ are successive integer intervals, that for all $j, E_{j} \cap \operatorname{supp} x_{k} \neq \emptyset$ for some $k$, and that $i_{p} \leq \max E_{q}$. Also let $I_{k}$ be the integer interval $\left[i_{k-1}+1, i_{k}\right]\left(i_{0} \equiv 0\right)$. Let $A=\left\{j: E_{j} \nsubseteq \subseteq I_{k}\right.$ for any $\left.k\right\}$ and $B=\{j: j \notin A\}$. For $j \in A$, set $H_{j}=\left\{k: I_{k} \subseteq E_{j}\right\}$ and $G_{j}=\left\{i_{k}: k \in H_{j}\right\}$. 
Then define $F_{j}=\left(E_{j} \cap\left\{i_{1}, \ldots, i_{p}\right\}\right) \backslash G_{j}$. Note that $F_{j}<G_{j}$ for all $j \in A$. If $j \in B$, set $G_{j}=E_{j} \cap\left\{i_{1}, \ldots, i_{p}\right\}$.

It follows from Lemma 3 that $\left(F_{j}\right)_{j \in A} \cup\left(G_{j}\right)_{j=1}^{q}$ is $\left(\mathcal{S}_{\beta}\right)^{2}$-admissible. Finally, let $J=\left\{k: k \notin \bigcup_{j \in A} H_{j}, I_{k} \cap\left(\bigcup E_{j}\right) \neq \emptyset\right\}$. Now

$$
\begin{aligned}
\sum_{j=1}^{q} \| & E_{j}\left(\sum_{k=1}^{p} a_{k} x_{k}\right)\left\|_{n}=\sum_{j=1}^{q}\right\| E_{j}\left(\sum_{j^{\prime} \in A} \sum_{k \in H_{j^{\prime}}} a_{k} x_{k}+\sum_{k \in J} a_{k} x_{k}\right) \|_{n} \\
& \leq \sum_{j=1}^{q}\left(\left\|E_{j}\left(\sum_{j^{\prime} \in A} \sum_{k \in H_{j^{\prime}}} a_{k} x_{k}\right)\right\|_{n}+\left\|E_{j}\left(\sum_{k \in J} a_{k} x_{k}\right)\right\|_{n}\right) \\
& =\sum_{j \in A}\left\|E_{j}\left(\sum_{k \in H_{j}} a_{k} x_{k}\right)\right\|_{n}+\sum_{j=1}^{q}\left\|E_{j}\left(\sum_{k \in J} a_{k} x_{k}\right)\right\|_{n} \\
& \leq \sum_{j \in A}\left\|\sum_{k \in H_{j}} a_{k} x_{k}\right\|_{n}+\sum_{k \in J}\left|a_{k}\right| \sum_{j=1}^{q}\left\|E_{j} x_{k}\right\|_{n} \\
& \leq \sum_{j \in A}\left\|\sum_{k \in H_{j}} a_{k} x_{k}\right\|_{n}+2 \sum_{k \in J}\left|a_{k}\right|\left\|x_{k}\right\|_{n+1} \\
& \leq 2\left(\sum_{j \in A}\left\|\sum_{k \in H_{j}} a_{k} e_{i_{k}}\right\|_{n}^{\prime}+\sum_{k \in J}\left|a_{k}\right|\right) \text { by the inductive hypothesis, } \\
& =2\left(\sum_{j \in A}\left\|G_{j}\left(\sum_{k=1}^{p} a_{k} e_{i_{k}}\right)\right\|_{n}^{\prime}+\sum_{k \in J}\left|a_{k}\right|\right) .
\end{aligned}
$$

If $k \in J$, then either $\left\{i_{k}\right\}=F_{j}$ for some $j \in A$ or $\left\{i_{k}\right\}=G_{j}$ for some $j \in B$. Therefore

$$
\sum_{k \in J}\left|a_{k}\right| \leq \sum_{j \in A}\left\|F_{j}\left(\sum_{k=1}^{p} a_{k} e_{i_{k}}\right)\right\|_{n}^{\prime}+\sum_{j \in B}\left\|G_{j}\left(\sum_{k=1}^{p} a_{k} e_{i_{k}}\right)\right\|_{n}^{\prime} .
$$

Hence

$$
\begin{gathered}
\sum_{j=1}^{q}\left\|E_{j}\left(\sum_{k=1}^{p} a_{k} x_{k}\right)\right\|_{n} \leq 2 \sum_{j \in A}\left\|G_{j}\left(\sum_{k=1}^{p} a_{k} e_{i_{k}}\right)\right\|_{n}^{\prime}+2 \sum_{j \in A}\left\|F_{j}\left(\sum_{k=1}^{p} a_{k} e_{i_{k}}\right)\right\|_{n}^{\prime} \\
\quad+2 \sum_{j \in B}\left\|G_{j}\left(\sum_{k=1}^{p} a_{k} e_{i_{k}}\right)\right\|_{n}^{\prime} \\
=2\left(\sum_{j \in A}\left\|F_{j}\left(\sum_{k=1}^{p} a_{k} e_{i_{k}}\right)\right\|_{n}^{\prime}+\sum_{j=1}^{q}\left\|G_{j}\left(\sum_{k=1}^{p} a_{k} e_{i_{k}}\right)\right\|_{n}^{\prime}\right) \\
\leq 4\left\|\sum_{k=1}^{p} a_{k} e_{i_{k}}\right\|_{n+1}^{\prime}, \text { as }\left(F_{j}\right)_{j \in A} \cup\left(G_{j}\right)_{j=1}^{q} \text { is }\left(\mathcal{S}_{\beta}\right)^{2} \text {-admissible. }
\end{gathered}
$$


Thus

$$
\frac{1}{2} \sum_{j=1}^{q}\left\|E_{j}\left(\sum_{k=1}^{p} a_{k} x_{k}\right)\right\|_{n} \leq 2\left\|\sum_{k=1}^{p} a_{k} e_{i_{k}}\right\|_{n+1}^{\prime}
$$

whenever $\left\{E_{1}, \ldots, E_{q}\right\}$ is $\mathcal{S}_{\beta}$-admissible. It follows that

$$
\left\|\sum_{k=1}^{p} a_{k} x_{k}\right\|_{n+1} \leq 2\left\|\sum_{k=1}^{p} a_{k} e_{i_{k}}\right\|_{n+1}^{\prime}
$$

This completes the induction.

Let $\alpha, \beta$ be countable ordinals. Define the families $\left(\mathcal{F}_{n}\right),\left(\mathcal{F}_{n}^{\prime}\right),\left(\mathcal{G}_{n}\right)$ and $\left(\mathcal{G}_{n}^{\prime}\right)$ inductively as follows: $\mathcal{F}_{0}=\mathcal{F}_{0}^{\prime}=\mathcal{S}_{\alpha}, \mathcal{G}_{1}=\mathcal{S}_{\beta}, \mathcal{G}_{1}^{\prime}=\left(\mathcal{S}_{\beta}\right)^{2}$, for all $n \in \mathbb{N}$,

$$
\mathcal{F}_{n+1}=\mathcal{S}_{\beta}\left[\mathcal{F}_{n}\right], \mathcal{F}_{n+1}^{\prime}=\left(\mathcal{S}_{\beta}\right)^{2}\left[\mathcal{F}_{n}^{\prime}\right], \mathcal{G}_{n+1}=\mathcal{S}_{\beta}\left[\mathcal{G}_{n}\right] \text {, and } \mathcal{G}_{n+1}^{\prime}=\left(\mathcal{S}_{\beta}\right)^{2}\left[\mathcal{G}_{n}^{\prime}\right] \text {. }
$$

It is easily verified that $\mathcal{G}_{n}\left[\mathcal{S}_{\alpha}\right]=\mathcal{F}_{n}, \mathcal{G}_{n}^{\prime}\left[\mathcal{S}_{\alpha}\right]=\mathcal{F}_{n}^{\prime}, \mathcal{G}_{n}\left[\mathcal{S}_{\beta}\right]=\mathcal{G}_{n+1}$ and $\mathcal{G}_{n}^{\prime}\left[\left(\mathcal{S}_{\beta}\right)^{2}\right]=\mathcal{G}_{n+1}^{\prime}$ for all $n \in \mathbb{N}$. For each $n \in \mathbb{N}$, denote the norms $\|\cdot\|_{\mathcal{F}_{n}}$ and $\|\cdot\|_{\mathcal{F}_{n}^{\prime}}$ by $\rho_{n}$ and $\rho_{n}^{\prime}$ respectively.

Proposition 5. For all $a \in c_{00}$ and all $n \in \mathbb{N} \cup\{0\},\|a\|_{\widetilde{T}} \geq \frac{1}{2^{n}} \rho_{n}(a)$.

Proof. The proof is by induction on $n$. The case $n=0$ is clearly true by definition. Suppose the result holds for some $n$. Let $E \in \mathcal{F}_{n+1}$. Then $E=\bigcup_{i=1}^{j} E_{i}$, where $E_{1}, \ldots, E_{j} \in \mathcal{F}_{n}, E_{1}<\ldots<E_{j}$, and $\left\{E_{1}, \ldots, E_{j}\right\}$ is $\mathcal{S}_{\beta}$-admissible. For any $a=$ $\left(a_{k}\right) \in c_{00}$

$$
\sum_{k \in E}\left|a_{k}\right|=\sum_{i=1}^{j} \sum_{k \in E_{i}}\left|a_{k}\right| \leq \sum_{i=1}^{j} \rho_{n}\left(E_{i} a\right) \leq 2^{n} \sum_{i=1}^{j}\left\|E_{i} a\right\|_{\widetilde{T}} \leq 2^{n+1}\|a\|_{\widetilde{T}} .
$$

Since $E \in \mathcal{F}_{n+1}$ is arbitrary, the result follows.

Proposition 6. For all $a \in c_{00}$ and all $n \in \mathbb{N} \cup\{0\}$,

$$
\|a\|_{\widetilde{T}} \leq \sum_{i=0}^{n} \frac{\rho_{i}^{\prime}(a)}{2^{i}}+\frac{1}{2^{n+1}} \sup \left\{\sum_{i=1}^{j}\left\|E_{i} a\right\|_{\widetilde{T}}:\left\{E_{1}, \ldots, E_{j}\right\} \mathcal{G}_{n+1}^{\prime} \text {-admissible }\right\} .
$$

Proof. The proof is by induction on $n$. The case $n=0$ follows immediately from the definition of $\|\cdot\|_{\tilde{T}}$. Assume the result holds for some $n$. Let $a \in c_{00}$. Suppose $\left\{E_{1}, \ldots, E_{j}\right\}$ is $\mathcal{G}_{n+1}^{\prime}$-admissible. Let $I=\left\{i:\left\|E_{i} a\right\|_{\widetilde{T}}=\rho_{0}^{\prime}\left(E_{i} a\right)\right\}$ and $J=$ $\{1,2, \ldots, j\} \backslash I$. For each $i \in I$, choose $D_{i} \subseteq E_{i}, D_{i} \in \mathcal{S}_{\alpha}$, such that $\rho_{0}^{\prime}\left(E_{i} a\right)=$ $\sum_{k \in D_{i}}\left|a_{k}\right|$. Now $D=\bigcup_{i \in I} D_{i} \in \mathcal{G}_{n+1}^{\prime}\left[\mathcal{S}_{\alpha}\right]=\mathcal{F}_{n+1}^{\prime}$. Hence

$$
\sum_{i \in I}\left\|E_{i} a\right\|_{\widetilde{T}}=\sum_{k \in D}\left|a_{k}\right| \leq \rho_{n+1}^{\prime}(a) .
$$

On the other hand, for each $i \in J$, there exist $\left(\mathcal{S}_{\beta}\right)^{2}$-admissible sets $\left\{E_{1}^{i}, \ldots, E_{k_{i}}^{i}\right\}$, $E_{1}^{i} \cup \ldots \cup E_{k_{i}}^{i} \subseteq E_{i}$ such that

$$
\left\|E_{i} a\right\|_{\widetilde{T}}=\frac{1}{2} \sum_{p=1}^{k_{i}}\left\|E_{p}^{i} a\right\|_{\widetilde{T}}
$$


Now

$$
\left\{\min E_{p}^{i}: i \in J, 1 \leq p \leq k_{i}\right\} \in \mathcal{G}_{n+1}^{\prime}\left[\left(\mathcal{S}_{\beta}\right)^{2}\right]=\mathcal{G}_{n+2}^{\prime} .
$$

Hence $\left(E_{p}^{i}\right)_{i \in J, 1 \leq p \leq k_{i}}$ is $\mathcal{G}_{n+2}^{\prime}$-admissible. Thus

$$
\begin{aligned}
\sum_{i \in J}\left\|E_{i} a\right\|_{\widetilde{T}} & =\frac{1}{2} \sum_{i \in J} \sum_{p=1}^{k_{i}}\left\|E_{p}^{i} a\right\|_{\widetilde{T}} \\
& \leq \frac{1}{2} \sup \left\{\sum_{i=1}^{\ell}\left\|F_{i} a\right\|_{\widetilde{T}}:\left\{F_{1}, \ldots, F_{\ell}\right\} \mathcal{G}_{n+2}^{\prime} \text {-admissible }\right\}
\end{aligned}
$$

From the inductive hypothesis and inequalities (11) and (2) we get

$$
\begin{aligned}
\|a\|_{\tilde{\widetilde{T}}} & \leq \sum_{i=0}^{n} \frac{\rho_{i}^{\prime}(a)}{2^{i}}+\frac{1}{2^{n+1}}\left(\rho_{n+1}^{\prime}(a)+\frac{1}{2} \sup \left\{\sum_{i=1}^{\ell}\left\|F_{i} a\right\|_{\widetilde{T}}\right\}\right) \\
& =\sum_{i=0}^{n+1} \frac{\rho_{i}^{\prime}(a)}{2^{i}}+\frac{1}{2^{n+2}} \sup \left\{\sum_{i=1}^{\ell}\left\|F_{i} a\right\|_{\tilde{T}}\right\},
\end{aligned}
$$

where both suprema are taken over all $\mathcal{G}_{n+2}^{\prime}$-admissible sets $\left\{F_{1}, \ldots, F_{\ell}\right\}$. This completes the induction.

Endow $[\mathbb{N}]^{<\infty}$ with the product topology inherited from $2^{\mathbb{N}}$. If $\mathcal{F}$ is a closed subset of $[\mathbb{N}]^{<\infty}$, let $\mathcal{F}^{\prime}$ be the set of all limit points of $\mathcal{F}$. Define a transfinite sequence of sets $\left(\mathcal{F}^{(\alpha)}\right)_{\alpha<\omega_{1}}$ as follows: $\mathcal{F}^{(0)}=\mathcal{F}, \mathcal{F}^{(\alpha+1)}=\left(\mathcal{F}^{(\alpha)}\right)^{\prime}$ for all $\alpha<\omega_{1}$; $\mathcal{F}^{(\alpha)}=\bigcap_{\beta<\alpha} \mathcal{F}^{(\beta)}$ if $\alpha$ is a countable limit ordinal.

Definition 7 ([1]). Let $\mathcal{F} \subseteq[\mathbb{N}]^{<\infty}$ be regular. Define $\iota(\mathcal{F})$ to be the unique countable ordinal $\alpha$ such that $\mathcal{F}^{(\alpha)}=\{\emptyset\}$.

Let $F \in \mathcal{M}[\mathcal{N}], F \neq \emptyset$, where $\mathcal{M}$ and $\mathcal{N}$ are regular families. There exists a largest $k \in F$ such that $F \cap[1, k] \in \mathcal{N}$. Set $F_{1}=F \cap[1, k]$. If $F_{1}, F_{2}, \ldots, F_{n-1}$ have been defined and $F \backslash \bigcup_{i=1}^{n-1} F_{i} \neq \emptyset$, set $F_{n}=\left(F \backslash \bigcup_{i=1}^{n-1} F_{i}\right) \cap\left[1, k^{\prime}\right]$, where $k^{\prime}$ is the largest integer in $F$ such that $\left(F \backslash \bigcup_{i=1}^{n-1} F_{i}\right) \cap\left[1, k^{\prime}\right] \in \mathcal{N}$. Since $F$ is finite, there exists an $n$ such that $F=\bigcup_{i=1}^{n} F_{i}$. Now $F \in \mathcal{M}[\mathcal{N}]$ implies that there exists an $\mathcal{M}$-admissible collection $\left\{G_{1}, \ldots, G_{m}\right\}$ such that $F=\bigcup_{j=1}^{m} G_{j}$ and $G_{j} \in \mathcal{N}$, $1 \leq j \leq m$. By the choice of $F_{i}$ and the fact that $\mathcal{N}$ is hereditary, it is easy to see that $\min G_{i} \leq \min F_{i}, 1 \leq i \leq n$. Thus $\left\{F_{1}, \ldots, F_{n}\right\}$ is $\mathcal{M}$-admissible, as $\mathcal{M}$ is spreading. We call $\left(F_{i}\right)_{i=1}^{n}$ the standard representation of $F$ (as an element of $\mathcal{M}[\mathcal{N}])$.

Remark 8. Suppose that $\left(F_{i}\right)_{i=1}^{n}$ and $\left(G_{i}\right)_{i=1}^{m}$ are the standard representations of $F$ and $G$ respectively. If $\ell, k \in \mathcal{N}$ are such that $F \cap[1, \ell]=G \cap[1, \ell]$ and $\max F_{k} \leq \ell$, then by construction, $F_{i}=G_{i}, 1 \leq i \leq k$.

Lemma 9. Let $\mathcal{M}, \mathcal{N} \subseteq[\mathbb{N}]^{<\infty}$ be regular. Suppose that $\iota(\mathcal{N})=\alpha$. Then

$$
(\mathcal{M}[\mathcal{N}])^{(\alpha)}=\left(\mathcal{M}^{(1)}\right)[\mathcal{N}]
$$


Proof. Let $F \in\left(\mathcal{M}^{(1)}\right)[\mathcal{N}]$. Then $F$ can be written as $F=\bigcup_{i=1}^{n} F_{i}$, where $F_{1}<$ $F_{2}<\ldots<F_{n}, F_{1}, \ldots, F_{n} \in \mathcal{N}$ and $\left\{\min F_{1}, \ldots, \min F_{n}\right\} \in \mathcal{M}^{(1)}$. In particular, there exists $k_{0}>\max F$ such that $\left\{\min F_{1}, \ldots, \min F_{n}, k\right\} \in \mathcal{M}$ for all $k \geq k_{0}$. Therefore,

$$
\text { for all } G \in \mathcal{N}, \min G \geq k_{0}, F \cup G \in \mathcal{M}[\mathcal{N}] \text {. }
$$

Note that as $\mathcal{N}$ is spreading,

$$
\iota\left(\left\{G \in \mathcal{N}: \min G \geq k_{0}\right\}\right)=\iota(\mathcal{N})=\alpha .
$$

From (3),

$$
\left(\left\{F \cup G: G \in \mathcal{N}, \min G \geq k_{0}\right\}\right)^{(\alpha)} \subseteq(\mathcal{M}[\mathcal{N}])^{(\alpha)} .
$$

But from (44), $F \in\left(\left\{F \cup G: G \in \mathcal{N}, \min G \geq k_{0}\right\}\right)^{(\alpha)}$. Hence $F \in(\mathcal{M}[\mathcal{N}])^{(\alpha)}$.

Conversely, we prove by induction that $(\mathcal{M}[\mathcal{N}])^{(\gamma)} \subseteq\left(\left(\mathcal{M}^{(1)}\right)[\mathcal{N}], \mathcal{N}^{(\gamma)}\right)$ for all $\gamma \leq \alpha$. The cases where $\gamma=0$ is clear.

Suppose the claim is true for some $\gamma<\alpha$. Let $F \in(\mathcal{M}[\mathcal{N}])^{(\gamma+1)}$ with standard representation $\left(F_{i}\right)_{i=1}^{n}$ as an element of $\mathcal{M}[\mathcal{N}]$. Choose a sequence $\left(G_{k}\right)$ in $(\mathcal{M}[\mathcal{N}])^{(\gamma)} \subseteq\left(\left(\mathcal{M}^{(1)}\right)[\mathcal{N}], \mathcal{N}^{(\gamma)}\right)$ that converges nontrivially to $F$. We may assume that $G_{k} \cap\left[1, \min F_{n}\right]=F \cap\left[1, \min F_{n}\right]$ for all $k$. Now we may write $G_{k}=P_{k} \cup Q_{k}$, where $P_{k}<Q_{k}, P_{k} \in\left(\mathcal{M}^{(1)}\right)[\mathcal{N}]$ and $Q_{k} \in \mathcal{N}^{(\gamma)}$. Let $P=\bigcup_{i=1}^{n-1} F_{i}$. Note that $P \in\left(\mathcal{M}^{(1)}\right)[\mathcal{N}]$. We consider two cases.

Case 1. There exists $k$ such that $\min F_{n} \in P_{k}$.

In this case, $P \cap\left[1, \max F_{n-1}\right]=F \cap\left[1, \max F_{n-1}\right]=G_{k} \cap\left[1, \max F_{n-1}\right]=$ $P_{k} \cap\left[1, \max F_{n-1}\right]$. It is clear that $\left(F_{i}\right)_{i=1}^{n-1}$ is the standard representation of $P$ as an element of $\left(\mathcal{M}^{(1)}\right)[\mathcal{N}]$. By Remark 8 the standard representation of $P_{k}$ as an element of $\left(\mathcal{M}^{(1)}\right)[\mathcal{N}]$ has the form $\left(F_{1}, \ldots, F_{n-1}, P_{k}^{n}, \ldots, P_{k}^{m}\right)$. In particular,

$$
\left\{\min F_{1}, \ldots, \min F_{n-1}, \min F_{n}\right\}=\left\{\min F_{1}, \ldots, \min F_{n-1}, \min P_{k}^{n}\right\} \in \mathcal{M}^{(1)} .
$$

Thus $F=\bigcup_{i=1}^{n} F_{i} \in\left(\mathcal{M}^{(1)}\right)[\mathcal{N}] \subseteq\left(\left(\mathcal{M}^{(1)}\right)[\mathcal{N}], \mathcal{N}^{(\gamma+1)}\right)$, as required.

Case 2. Suppose $\min F_{n} \notin P_{k}$ for all $k \in \mathbb{N}$.

In this case, $G_{k} \cap\left[\min F_{n}, \infty\right) \subseteq Q_{k}$ for all $k$. Hence $G_{k} \cap\left[\min F_{n}, \infty\right) \in \mathcal{N}^{(\gamma)}$ for all $k$. Furthermore, $G_{k} \cap\left[\min F_{n}, \infty\right)$ converges to $F \cap\left[\min F_{n}, \infty\right)=F_{n}$ nontrivially. Thus $F_{n} \in \mathcal{N}^{(\gamma+1)}$. Therefore $F=P \cup F_{n} \in\left(\left(\mathcal{M}^{(1)}\right)[\mathcal{N}], \mathcal{N}^{(\gamma+1)}\right)$, as required.

Suppose $\gamma \leq \alpha$ is a limit ordinal and the result holds for all $\eta<\gamma$. Let $F \in(\mathcal{M}[\mathcal{N}])^{(\gamma)}$ have standard representation $\left(F_{i}\right)_{i=1}^{n}$ as an element of $\mathcal{M}[\mathcal{N}]$. By the inductive hypothesis, for each $\eta<\gamma, F=P_{\eta} \cup Q_{\eta}$, where $P_{\eta}<Q_{\eta}$, $P_{\eta} \in\left(\mathcal{M}^{(1)}\right)[\mathcal{N}]$ and $Q_{\eta} \in \mathcal{N}^{(\eta)}$. By the argument in Case 1 above, if there exists $\eta$ such that $\min F_{n} \in P_{\eta}$, then $F \in\left(\mathcal{M}^{(1)}\right)[\mathcal{N}] \subseteq\left(\left(\mathcal{M}^{(1)}\right)[\mathcal{N}], \mathcal{N}^{(\eta)}\right)$. Otherwise, $F_{n} \subseteq Q_{\eta} \in \mathcal{N}^{(\eta)}$ for all $\eta<\gamma$. Hence $F=\left(\bigcup_{i=1}^{n-1} F_{i}\right) \cup F_{n} \in\left(\left(\mathcal{M}^{(1)}\right)[\mathcal{N}], \mathcal{N}^{(\gamma)}\right)$. This completes the induction.

Proposition 10. Let $\mathcal{M}, \mathcal{N} \subseteq[\mathbb{N}]^{<\infty}$ be regular. Suppose that $\iota(\mathcal{N})=\alpha$. Then for all $\beta<\omega_{1},(\mathcal{M}[\mathcal{N}])^{(\alpha \cdot \beta)}=\left(\mathcal{M}^{(\beta)}\right)[\mathcal{N}]$. 
Proof. The proof is by induction on $\beta$. The case $\beta=0$ is clear. Suppose the result is true for some $\beta$. Then

$$
\begin{aligned}
(\mathcal{M}[\mathcal{N}])^{(\alpha \cdot(\beta+1))} & =(\mathcal{M}[\mathcal{N}])^{(\alpha \cdot \beta+\alpha)} \\
& =\left((\mathcal{M}[\mathcal{N}])^{(\alpha \cdot \beta)}\right)^{(\alpha)} \\
& =\left(\left(\mathcal{M}^{(\beta)}\right)[\mathcal{N}]\right)^{(\alpha)} \text { by the inductive hypothesis, } \\
& =\left(\left(\mathcal{M}^{(\beta)}\right)^{(1)}[\mathcal{N}]\right) \text { by Lemma } 9 \\
& =\left(\mathcal{M}^{(\beta+1)}\right)[\mathcal{N}] .
\end{aligned}
$$

Suppose the proposition is true for all $\beta<\beta_{0}$, where $\beta_{0}<\omega_{1}$ is some limit ordinal. Clearly,

$$
\left(\mathcal{M}^{\left(\beta_{0}\right)}\right)[\mathcal{N}] \subseteq \bigcap_{\beta<\beta_{0}}\left(\mathcal{M}^{(\beta)}\right)[\mathcal{N}]=\bigcap_{\beta<\beta_{0}}(\mathcal{M}[\mathcal{N}])^{(\alpha \cdot \beta)}=(\mathcal{M}[\mathcal{N}])^{\left(\alpha \cdot \beta_{0}\right)} .
$$

On the other hand, let $F \in \bigcap_{\beta<\beta_{0}}\left(\mathcal{M}^{(\beta)}\right)[\mathcal{N}]$ have standard representation $\left(F_{i}\right)_{i=1}^{n}$ as an element of $\mathcal{M}[\mathcal{N}]$. It is clear that $\left(F_{i}\right)_{i=1}^{n}$ is also the standard representation of $F$ as an element of $\left(\mathcal{M}^{(\beta)}\right)[\mathcal{N}]$ for any $\beta<\beta_{0}$. In particular, $\left\{\min F_{i}: 1 \leq i \leq n\right\} \in$ $\mathcal{M}^{(\beta)}$ for all $\beta<\beta_{0}$. Hence $\left\{\min F_{i}: 1 \leq i \leq n\right\} \in \mathcal{M}^{\left(\beta_{0}\right)}$. It follows that $F \in$ $\left(\mathcal{M}^{\left(\beta_{0}\right)}\right)[\mathcal{N}]$. This completes the proof.

It is well known that $\iota\left(\mathcal{S}_{\gamma}\right)=\omega^{\gamma}$ for all $\gamma<\omega_{1}$ ([1, Proposition 4.10]). The indices of $\mathcal{F}_{n}$ and $\mathcal{F}_{n}^{\prime}$ can be computed readily with the help of Proposition 10.

Corollary 11. $\iota\left(\mathcal{F}_{n}\right)=\omega^{\alpha+\beta \cdot n}$ and $\iota\left(\mathcal{F}_{n}^{\prime}\right)=\omega^{\alpha+\beta \cdot n} \cdot 2$.

Before proceeding further, let us recall the relevant terminology concerning trees. A tree on a set $X$ is a subset $T$ of $\bigcup_{n=1}^{\infty} X^{n}$ such that $\left(x_{1}, \ldots, x_{n}\right) \in T$ whenever $n \in$ $\mathbb{N}$ and $\left(x_{1}, \ldots, x_{n+1}\right) \in T$. These are the only kind of trees we will consider. A tree $T$ is well-founded if there is no infinite sequence $\left(x_{n}\right)$ in $X$ such that $\left(x_{1}, \ldots, x_{n}\right) \in T$ for all $n$. Given a well-founded tree $T$, we define the derived tree $D(T)$ to be the set of all $\left(x_{1}, \ldots, x_{n}\right) \in T$ such that $\left(x_{1}, \ldots, x_{n}, x\right) \in T$ for some $x \in X$. Inductively, we let $D^{0}(T)=T, D^{\alpha+1}(T)=D\left(D^{\alpha}(T)\right)$, and $D^{\alpha}(T)=\bigcap_{\beta<\alpha} D^{\beta}(T)$ if $\alpha$ is a limit ordinal. The order of a well-founded tree $T$ is the smallest ordinal $o(T)$ such that $D^{o(T)}(T)=\emptyset$. If $E$ is a Banach space and $1 \leq K<\infty$, an $\ell^{1}-K$ tree on $E$ is a tree $T$ on $S(E)=\{x \in E:\|x\|=1\}$ such that $\left\|\sum_{i=1}^{n} a_{i} x_{i}\right\| \geq K^{-1} \sum_{i=1}^{n}\left|a_{i}\right|$ whenever $\left(x_{1}, \ldots, x_{n}\right) \in T$ and $\left(a_{i}\right) \subseteq \mathbb{R}$. If $E$ has a basis $\left(e_{i}\right)$, a block tree on $E$ is a tree $T$ on $E$ so that every $\left(x_{1}, \ldots, x_{n}\right) \in T$ is a finite block basis of $\left(e_{i}\right)$. An $\ell^{1}-K$-block tree on $E$ is a block tree that is also an $\ell^{1}-K$ tree. The index $I(E, K)$ is defined to be $\sup \left\{o(T): T\right.$ is an $\ell^{1}-K$ tree on $\left.E\right\}$. If $E$ has a basis $\left(e_{i}\right)$, the index $I_{b}(E, K)$ is defined similarly, with the supremum taken over all $\ell^{1}-K$-block trees. The Bourgain $\ell^{1}$-index of $E$ is the ordinal $I(E)=\sup \{I(E, K): 1 \leq K<\infty\}$. The index $I_{b}(E)$ is defined similarly. Bourgain proved that if $E$ is a separable Banach space not containing a copy of $\ell^{1}$, then $I(E)<\omega_{1}[4$. More on these and related indices can be found in $[8$ and $[3]$.

Proposition 12. Let $T$ be a well-founded block tree on some basis $\left(e_{i}\right)$. Define

$$
\mathcal{F}(T)=\left\{\left\{\max \operatorname{supp} x_{i}: i=1, \ldots, n\right\}:\left(x_{1}, x_{2}, \ldots, x_{n}\right) \in T\right\}
$$


and

$\mathcal{G}(T)=\{G: \exists F \in \mathcal{F}(T), f: \mathbb{N} \rightarrow \mathbb{N}$ strictly increasing, such that $G \subseteq f(F)\}$.

Then $\iota(\mathcal{G}(T)) \geq o(T)$.

Proof. Let $\xi=o(T)$. The proof is by induction on $\xi$. If $o(T)=1$, then $\mathcal{G}(T) \supseteq$ $\{\{k\}: k \geq n\}$ for some $n \in \mathbb{N}$. Therefore $(\mathcal{G}(T))^{(1)} \supseteq\{\emptyset\}$ and hence $\iota(\mathcal{G}(T)) \geq 1=$ $o(T)$.

Suppose the proposition holds for some $\xi<\omega_{1}$. Let $T$ be a well-founded block tree with $o(T)=\xi+1$ such that $\mathcal{G}(T)$ is compact. For each $(x) \in T$, let

$$
T_{x}=\bigcup_{n=1}^{\infty}\left\{\left(x_{1}, \ldots, x_{n}\right):\left(x, x_{1}, \ldots, x_{n}\right) \in T\right\} .
$$

According to [4, Proposition 4], $o(T)=\sup _{(x) \in T}\left\{o\left(T_{x}\right)+1\right\}$. Therefore, there exists $\left(x_{0}\right) \in T$ such that $o\left(T_{x_{0}}\right)=\xi$. By the inductive hypothesis, $\iota\left(\mathcal{G}\left(T_{x_{0}}\right)\right) \geq \xi$. Let $k_{0}=\max \operatorname{supp} x_{0}$. Then $G \in \mathcal{G}\left(T_{x_{0}}\right)$ implies $\left\{k_{0}\right\} \cup G \in \mathcal{G}(T)$. Thus $\left\{k_{0}\right\} \in$ $(\mathcal{G}(T))^{(\xi)}$. Since $(\mathcal{G}(T))^{(\xi)}$ is spreading, $\{k\} \in(\mathcal{G}(T))^{(\xi)}$ for all $k \geq k_{0}$. It follows that $\emptyset \in(\mathcal{G}(T))^{(\xi+1)}$. Hence $\iota(\mathcal{G}(T)) \geq \xi+1=o(T)$.

Suppose $o(T)=\xi_{0}$, where $\xi_{0}$ is a countable limit ordinal and the proposition holds for all $\xi<\xi_{0}$. Since $o(T)>\xi$ for all $\xi<\xi_{0}$, by the inductive hypothesis, $\iota(\mathcal{G}(T))>\xi$ for all $\xi<\xi_{0}$. Hence $\iota(\mathcal{G}(T)) \geq \xi_{0}=o(T)$. This completes the induction.

If $\left(x_{k}\right)_{k=1}^{n}$ and $\left(y_{k}\right)_{k=1}^{n}$ are sequences in possibly different normed spaces and $0<K<\infty$, we write $\left(x_{k}\right)_{k=1}^{n} \stackrel{K}{\succeq}\left(y_{k}\right)_{k=1}^{n}$ to mean $K\left\|\sum_{k=1}^{n} a_{k} x_{k}\right\| \geq\left\|\sum_{k=1}^{n} a_{k} y_{k}\right\|$ for all $\left(a_{k}\right) \subseteq \mathbb{R}$.

Theorem 13. $I\left(\tilde{T}_{\alpha, \beta}\right)=I_{b}\left(\widetilde{T}_{\alpha, \beta}\right)=\omega^{\alpha+\beta \cdot \omega}$.

Proof. If $I_{b}\left(\widetilde{T}_{\alpha, \beta}\right)>\omega^{\alpha+\beta \cdot \omega}$, then $I_{b}\left(\widetilde{T}_{\alpha, \beta}, K\right)>\omega^{\alpha+\beta \cdot \omega}$ for some $K>1$. Hence there exists an $\ell^{1}-K$-block tree $T$ on $\widetilde{T}_{\alpha, \beta}$ such that $o(T)=\xi>\omega^{\alpha+\beta \cdot \omega}$. Given $F \in \mathcal{F}(T)$, there exists $\left(x_{1}, x_{2}, \ldots, x_{n}\right) \in T$ such that $F=\left\{\max \operatorname{supp} x_{i}\right\}_{i=1}^{n}$. According to Proposition 4. $\left(e_{k}\right)_{k \in F} \succeq\left(x_{1}, x_{2}, \ldots, x_{n}\right)$, where $\left(e_{k}\right)_{k=1}^{\infty}$ is the unit vector basis of $\widetilde{\widetilde{T}}_{\alpha, \beta}$. Since $\left(x_{1}, x_{2}, \ldots, x_{n}\right) \in T,\left(x_{1}, x_{2}, \ldots, x_{n}\right) \stackrel{K}{\succeq}^{1} \ell^{1}(|F|)$-basis. Therefore, $\left(e_{k}\right)_{k \in F} \stackrel{2 K}{\succeq} \ell^{1}(|F|)$-basis for all $F \in \mathcal{F}(T)$. Since it is clear that $\left\|\sum a_{k} e_{f(k)}\right\|_{\widetilde{T}} \geq\left\|\sum a_{k} e_{k}\right\|_{\widetilde{T}}$ for all $\left(a_{k}\right) \in c_{00}$ whenever $f: \mathbb{N} \rightarrow \mathbb{N}$ is strictly increasing, it follows that $\left(e_{k}\right)_{k \in G} \stackrel{2 K}{\succeq} \ell^{1}(|G|)$-basis for all $G \in \mathcal{G}(T)$. By Proposition 12] $(\mathcal{G}(T))^{\left(\omega^{\alpha+\beta \cdot \omega}+1\right)} \neq \emptyset$. Thus by [7, Corollary 1.2], there exists $L \in[\mathbb{N}]$ such that $\mathcal{S}_{\alpha+\beta \cdot \omega} \cap[L]^{<\infty} \subseteq \mathcal{G}(T)$. Hence, for all $\left(a_{k}\right) \in c_{00}$ and all $G \in \mathcal{S}_{\alpha+\beta \cdot \omega} \cap[L]^{<\infty}$,

$$
\left\|\sum_{k \in G} a_{k} e_{k}\right\|_{\widetilde{\widetilde{T}}} \geq \frac{1}{2 K} \sum_{k \in G}\left|a_{k}\right| .
$$

Choose $m \in \mathbb{N}$ such that $2^{m}>2 K$. According to Corollary 11, $\iota\left(\mathcal{F}_{i}^{\prime}\right)=\omega^{\alpha+\beta \cdot i} \cdot 2$ for all $i=1,2, \ldots, m$. Applying [7, Corollary 1.2], we obtain $M \in[L]$ such that $\mathcal{F}_{i}^{\prime} \cap[M]^{<\infty} \subseteq \mathcal{S}_{\alpha+\beta \cdot m+1}$ for all $i=1,2, \ldots, m$. By [11] Proposition 3.6], there exists 
$F \in \mathcal{S}_{\alpha+\beta \cdot \omega}(M) \subseteq \mathcal{S}_{\alpha+\beta \cdot \omega} \cap[M]^{<\infty}$ and $\left(a_{j}\right)_{j \in F} \subseteq \mathbb{R}^{+}$such that $\sum a_{j}=1$ and if $G \subseteq F$ with $G \in \mathcal{S}_{\alpha+\beta \cdot m+1}$, then $\sum_{j \in G} a_{j}<\frac{1}{8 K}$. Consider $x=\sum_{j \in F} a_{j} e_{j} \in \widetilde{\widetilde{T}}_{\alpha, \beta}$. If $1 \leq i \leq m$ and $I \in \mathcal{F}_{i}^{\prime}$, then $I \cap F \in \mathcal{F}_{i}^{\prime} \cap[M]^{<\infty} \subseteq \mathcal{S}_{\alpha+\beta \cdot m+1}$. Hence $\sigma_{I}(x)=$ $\sigma_{I \cap F}(x)<\frac{1}{8 K}$. It follows that $\rho_{i}^{\prime}(x) \leq \frac{1}{8 K}$ for $1 \leq i \leq m$. By Proposition 6

$$
\begin{aligned}
\|x\|_{\widetilde{T}} & \leq \sum_{i=0}^{m} \frac{\rho_{i}^{\prime}(x)}{2^{i}}+\frac{1}{2^{m+1}} \sup \left\{\sum_{i=1}^{j}\left\|E_{i} x\right\|_{\widetilde{T}}:\left\{E_{1}, \ldots, E_{j}\right\} \mathcal{G}_{m+1}^{\prime} \text {-admissible }\right\} \\
& \leq \sum_{i=0}^{m} \frac{\frac{1}{8 K}}{2^{i}}+\frac{1}{2^{m+1}}\|x\|_{\ell^{1}}<\frac{1}{2 K}
\end{aligned}
$$

contrary to (5). This proves that $I_{b}\left(\widetilde{T}_{\alpha, \beta}\right) \leq \omega^{\alpha+\beta \cdot \omega}$. On the other hand, according to Proposition 5, for any $n \in \mathbb{N},\|a\|_{\widetilde{T}} \geq \frac{1}{2^{n}}\|a\|_{\mathcal{F}_{n}}$ for any $a \in c_{00}$. By Corollary [1], $\iota\left(\mathcal{F}_{n}\right)=\omega^{\alpha+\beta \cdot n}$. Therefore, there exists an $\ell^{1}-2^{n}$-block basis tree $T_{n}$ on $\tilde{T}_{\alpha, \beta}$ with $o\left(T_{n}\right)=\omega^{\alpha+\beta \cdot n}$. Hence $I_{b}\left(\tilde{T}_{\alpha, \beta}, 2^{n}\right) \geq \omega^{\alpha+\beta \cdot n}$. Thus $I_{b}\left(\widetilde{T}_{\alpha, \beta}\right)=\sup _{K} I_{b}\left(\tilde{T}_{\alpha, \beta}, K\right) \geq \omega^{\alpha+\beta \cdot \omega}$. We conclude that $I_{b}\left(\widetilde{T}_{\alpha, \beta}\right)=\omega^{\alpha+\beta \cdot \omega}$. As $I\left(\widetilde{T}_{\alpha, \beta}\right) \geq I_{b}\left(\widetilde{T}_{\alpha, \beta}\right) \geq \omega^{\omega}$, it follows from [8, Corollary 5.13] that $I\left(\widetilde{T}_{\alpha, \beta}\right)=$ $I_{b}\left(\tilde{T}_{\alpha, \beta}\right)$.

For the final corollary, recall that the Schreier space $X_{\alpha}, \alpha<\omega_{1}$, is the completion of $c_{00}$ with respect to the norm $\|\cdot\|_{\mathcal{S}_{\alpha}}$.

Corollary 14. Suppose $\alpha$ is a countable ordinal whose Cantor normal form is $\omega^{\alpha_{1}} \cdot m_{1}+\ldots+\omega^{\alpha_{k}} \cdot m_{k}$. If $\alpha_{k}$ is not a limit ordinal, then there exists a Banach space $X$ such that $I(X)=\omega^{\alpha}$.

Proof. If $\alpha_{k}=0$, then $\alpha$ is a successor ordinal and $\iota\left(X_{\alpha-1}\right)=\omega^{\alpha}([3])$. If $\alpha_{k}$ is a successor ordinal, let $\gamma=\omega^{\alpha_{1}} \cdot m_{1}+\ldots+\omega^{\alpha_{k}} \cdot\left(m_{k}-1\right)$ and $\eta=\omega^{\alpha_{k}-1}$. By Theorem 13 I $I\left(\tilde{T}_{\gamma, \eta}\right)=\omega^{\gamma+\eta \cdot \omega}=\omega^{\alpha}$.

Remark 15. The following analog of Proposition 4 for the space $X_{\alpha}, \alpha<\omega_{1}$, holds obviously: If $\left(x_{i}\right)_{i=1}^{p}$ is a normalized block basis of the unit vector basis $\left(e_{k}\right)$ of $X_{\alpha}$ and $k_{i}=\max \operatorname{supp} x_{i}, 1 \leq i \leq p$, then $\left\|\sum_{i=1}^{p} a_{i} x_{i}\right\| \leq\left\|\sum_{i=1}^{p} a_{i} e_{k_{i}}\right\|$ for all $\left(a_{i}\right) \in c_{00}$. Therefore the arguments in Proposition 12 and Theorem 13 can be used to compute $I_{b}\left(X_{\alpha}\right)$ (with respect to the basis $\left(e_{k}\right)$ ).

\section{REFERENCES}

[1] D. E. Alspach and S. Argyros, Complexity of weakly null sequences, Diss. Math., 321 (1992), 1-44. MR 93j:46014

[2] S. A. Argyros, S. Mercourakis and A. Tsarpalias, Convex unconditionality and summability of weakly null sequences, Israel J. Math. 107 (1998), 157-193. MR 99m:46021

[3] D. E. Alspach, R. Judd and E. Odell, The Szlenk index and local $\ell_{1}$-indices, preprint.

[4] J. Bourgain, On convergent sequences of continuous functions, Bull. Soc. Math. Bel., 32 (1980), 235-249. MR 84e:46018

[5] P. G. Casazza, W. B. Johnson and L. Tzafriri, On Tsirelson's space, Israel J. Math. 47 (1984), 81-98. MR 85m:46013

[6] T. Figiel and W. B. Johnson, A uniformly convex Banach space which contains no $\ell_{p}$, Compositio Math. 29 (1974), 179-190. MR 50:8011 
[7] I. Gasparis, A dichotomy theorem for subsets of the power set of the natural numbers, Proc. Amer. Math. Soc. 129 (2001), 759-764. MR 2001f:03089

[8] R. Judd, E. Odell, Concerning the Bourgain $\ell_{1}$ index of a Banach space, Israel J. Math. 108 (1998), 145-171. MR 2000k:46013

[9] P. Kiriakouli, Characterizations of spreading models of $\ell_{1}$, Comment. Math. Univ. Carolinae, 41(2000), 79-95. MR 2001e:46040

[10] D. H. Leung and W.-K. Tang, The Bourgain $\ell^{1}$-indices of mixed Tsirelson spaces, in preparation.

[11] Edward Odell, Nicole Tomczak-Jaegermann, and Roy Wagner, Proximity to $\ell_{1}$ and distortion in asymptotic $\ell_{1}$ spaces, Journal of Functional Analysis 150(1997), 101-145. MR 2000c:46027

[12] B. S. Tsirelson, Not every Banach space contains an embedding of $\ell_{p}$ or $c_{0}$, Functional Anal. Appl. 8 (1974), 138-141.

Department of Mathematics, National University of Singapore, Singapore 117543

E-mail address: matlhh@nus.edu.sg

Mathematics and Mathematics Education, National Institute of Education, Nanyang Technological University, 1 Nanyang Walk, Singapore 637616

E-mail address: wktang@nie.edu.sg 